\title{
IRREDUCIBLE TENSOR ANALYSIS OF SUM- AND DIFFERENCE-FREQUENCY GENERATION IN PARTIALLY ORIENTED SAMPLES
}

\author{
Bernhard DICK \\ Max-Planck-Institut fü Biophysikalische Chemie, Abreilung Laserphysik, D-j400 Götringen, FRG
}

Received 10 December 1985

\begin{abstract}
The macroscopic non-linear optical susceptibility $x^{(?)}$ for a system of partially oriented molecules is uritten as the ensemble average of molecular hyperpolarizability tensors $\beta$. The orientational distribution of these molecules is described by a probability function expanded in Wigner matrices. For a roiationally invariant system $x^{r}$ has seven non-vanishing components, and a method is cutlined to determine these by sum-or difference-frequency mixing experiments. The method could be applied to electrically poled samples, adsorbztes, liquid crystals, and membranes to determine several components of the molecular hyperpolarizability tensor, or to extract information about the orientational distribution.
\end{abstract}

\section{Introduction}

The interest in optical non-linear properties of organic materials is rapidly increasing Depending on structure and electronic resonances the second- and third-order non-linear susceptibilities $x^{(2)}$ and $x^{(3)}$ of such substances can be extremely large, exceedirg those of commonly used inorganic materials by several orders of magnitude [1,2]. These substances are therefore under discussion as possible media in frequency converters [3,4], modulators [5,6], non-linear waveguides [7], and other non-linear optical devices. These susceptibilities are usually measured by secord-harmonic [8-13] and third-harmonic [14-17] generation.

The dipolar part of the non-linear susceptibility $x^{(2)}$ vanishes in centrosymmetric media [18]. Its measurement and use requires some degree of orientation of the active molecules in the sample As a consequence, molecules with large second-order hyperpolarizability $\beta$ cannot be used in crystal form when these are centrosymmetric, as in the case of $p$-nitro-aniline [19]. On the other hand, $\beta$ cannot be measured directly in isotropically arranged sampies unless the molecuies are chiral and two light fields of different frequencies are mixed [20].

To obtain a maximum of information about all tensor components of $\beta$ the molecules have to be rigorously oriented, using the neat crystal if it is non-centrosymmetric or by doping them into a non-centrosymmetric host crystal lattice. As an alternative a centrosymmetric host material can be employed and the degeneracy of the two oppositely oriented sublattices of the guest molecules can be lifted with a dc electric field [21,22]. In this case the electronic resonant part of $\boldsymbol{B}$ can be studied virtually without interferences from the field-induced non-resonant $x^{(2)}$ of the host material. While all tensor elements could in principle be studied, the mixed crystal method is restricted to cases where an appropriate host material can be found. It is certainly not useful for routine measurements.

An alternative technique exists in the method of field-induced second-harmonic generation [23-27] developed mainly by Levine and Bethea [28-37] and Oudar and co-workers [38-42]. However, this method yields only one molecular parameter as an average of all tensor elements. In addition, the induced $x^{(\text {) }}$ contains not only contributions from the molecular hyperpolarizability $\beta$ via orientation of the molecular dipoles, but also new electronic contributions resulting from the molecular third-order hyperpolarizability 
$\gamma$ [43]. In cases where rotational diffusion is very slow the two effects can be separated by measuring the generated second-harmonic light before and just after the dc field is switched off [31].

In this paper we investigate the non-linear second-order response of uniaxially oriented samples. With respect to the degree of orientation this case is intermediate between the crystal and a random isotropic distribution. The sample is characterized by one axis around which it is rotationally invariant, and which lies in the direction of the averaged dipole moment. Such situations are frequently encountered in adsorbate systems on non-crystalline substrates $[44,45]$. The non-vanishing $x^{(2)}$ of surface layers has been investigated via second-harmonic generation by several groups [44-52] and the rotational invariance around the surface normal was found to hold in many cases. Oriented dipole layers are also known in many biological systems, especially membranes.

Unaxially oriented samples can be generated in the laboratory by poling of polymer matrices with static electric fields. When the sample is first heated and then cooled below the glas temperature while applying the de field the poling will be persistent [53,54].

Our main interest is to elucidate the relation between the macroscopic susceptibility $x^{(2)}$, the microscopic hyperpolarizability $\beta$ and the distribution function of the molecular orientation. The results are applicable to:

(a) Determination of molecular tensor elements of $\beta$ in cases where the molecular orientation is known, e.g- in poled polymer matrices.

(b) Determination of molecular orientation in cases where $\beta$ is known.

Both applications could be combined by studying a molecule first in a poled polymer matrix and subsequentiy adsorbed on a surface or doped as a probe into liquid crystals or membranes.

In section 2 we will briefly discuss the symmetry properties of $x^{(2)}$ in unaxially oriented systems. It will be convenient to use both cartesian and spherical coordinates. The latter allow a straightforward construction of the rotational invariants $[55,56]$. The third section is devoted to the discussion of the orientation distribution function which is used to connect the macroscopic susceptibility $x^{(2)}$ with the molecular hyperpolarizability $\beta$. In sections 4 and 5 examples are presented which demonstrate the application of the method to several situations of interest. Section 6 shows how the macroscopic quantities required for the analysis can be obtained by polarized sum- and difference-frequency generation.

\section{$2 . x^{(2)}$ in rotationally invariant systems}

Two light beams represented by classical c-number fields:

$$
\tilde{E}_{j}=\left(E_{j} \exp \left[-\mathrm{i}\left(\omega_{j} r-k_{j}-r\right)\right] \div \mathrm{c.c} ., j=1,2,\right.
$$

are incident on a non-linear medium, where they interact via the second-order susceptibility $\chi^{(2)}$ to form a non-linear polarization:

$$
\mathscr{P}=\mathrm{x}^{(2)}:\left(\mathscr{E}_{1}+\mathscr{E}_{2}\right)^{2}
$$

This polarization has Fourier components, among others, rotating with the sum frequency $\omega_{1}+\omega_{2}$ and the difference frequency $\omega_{2}-\omega_{1}$ - The expression for the sum frequency is:

$$
\mathscr{P}\left(\omega_{1} \div \omega_{2}\right)=P\left(\omega_{1}+\omega_{2}\right) \exp \left[i\left(k_{1}+k_{2}\right) \cdot r-i\left(\omega_{1}+\omega_{2}\right) t\right]
$$

with the amplitude vector given by:

$$
P\left(\omega_{1}+\omega_{2}\right)=x^{(2)}\left(\omega_{1}, \omega_{2}\right): E_{1} E_{2}
$$


The expression for the difference frequency has $\omega_{1}$ replaced by $-\omega_{1}$ and $E_{1}$ by $E_{1}^{*}$. In this and sections 3 and 4 we are concened with the tensor properties expressed in eq. (4). The wave character of the non-linear polarization as given in eq. (3) will be dealt, with in section 6 . From now on we drop all frequency arguments since the discussion is equally valid for sum- and difference-frequency generation.

Eq. (4) is a relation between a third-rank tensor $x^{(2)}$ and three first-rank tensors $E_{1}, E_{2}$, and $P$. In cartesian coordinates it has the form:

$$
P_{i}=\sum_{j k} x_{i j k} E_{1 j} E_{2 k}, \quad i j k \in\{X, Y, Z\}
$$

We take the laboratory $Z$ axis as the dipolar axis of the sample around which it is supposed to be rotationally invariant. For a surface layer this is the surface normal, for an electrically oriented sample it is the direction of the applied de field. Rotation of the sample around $Z$ by an arbitrary angle $\varphi$ transforms the cartesian components of a vector according to:

$$
X^{\prime}=X \cos \varphi-Y \sin \varphi, \quad Y^{\prime}=X \sin \varphi+Y r \cos \varphi, \quad Z^{\prime}=Z
$$

The components of $x^{(2)}$ will transform like the product of the corresponding three coordinates. Of the products of two coordinates the expressions $Z_{1} Z_{2}, X_{1} X_{2}+Y_{1} Y_{2}$ and $X_{1} Y_{2}-Y_{1} X_{2}$ are directly seen to be rotationally invariant. The invariant products of three coordinates are found by multiplication of these expressions with a further $Z$ component Since the tensor is required to be invariant as a whole, each component has to be invariant. This leads to the conditions $X_{1} X_{2}-Y_{1} Y_{2}=0$ and $X_{1} Y_{2}+Y_{1} X_{2}=0$ for each pair of indices in $X$. The seven non-vanishing ir ivariants of $\chi^{(2)}$ are:

$$
\begin{aligned}
& x_{Z Z Z}, x_{Z X X}=x_{Z Y Y}, x_{X Z X}=x_{Y Z Y}, x_{X X Z}=x_{Y Y Z}, \\
& x_{X Y Z}=-x_{Y X Z}, x_{X Z Y}=-x_{Y Z X}, x_{z} x_{Y Y}=-x_{Z Y X}
\end{aligned}
$$

If in addition to the rotation axis a mirror plane containing the $Z$ axis exists, the three invariants in (7b) will vanish. In the special case of second-harmionic generation the tensor is symmetric in its last two indices, leading to $\chi_{x z X}=x_{x \times z}, x_{x y z}=\chi_{x z y}$, ancl $\chi_{z x y}=0$. There are seven measurable parameters in the general case of sum- or difference-frequejucy generation and four in the case of second-harmonic generation. They reduce to four and three, respestively, in the presence of a mirror plane.

Table 1

Reduction of the $n$th rank cartesian tensors $R^{*}$ into irieducible representations of the rotation group $T^{l}$. Each $T^{\prime}$ of $R^{*}$ will produce three contributions $T^{l-1}, T^{l}, T^{t+1}$ for $R^{*+1}$. The $T_{0}^{l}$ :are the rotational invariants, the $T_{0}^{0}$ are the isotropic invariants

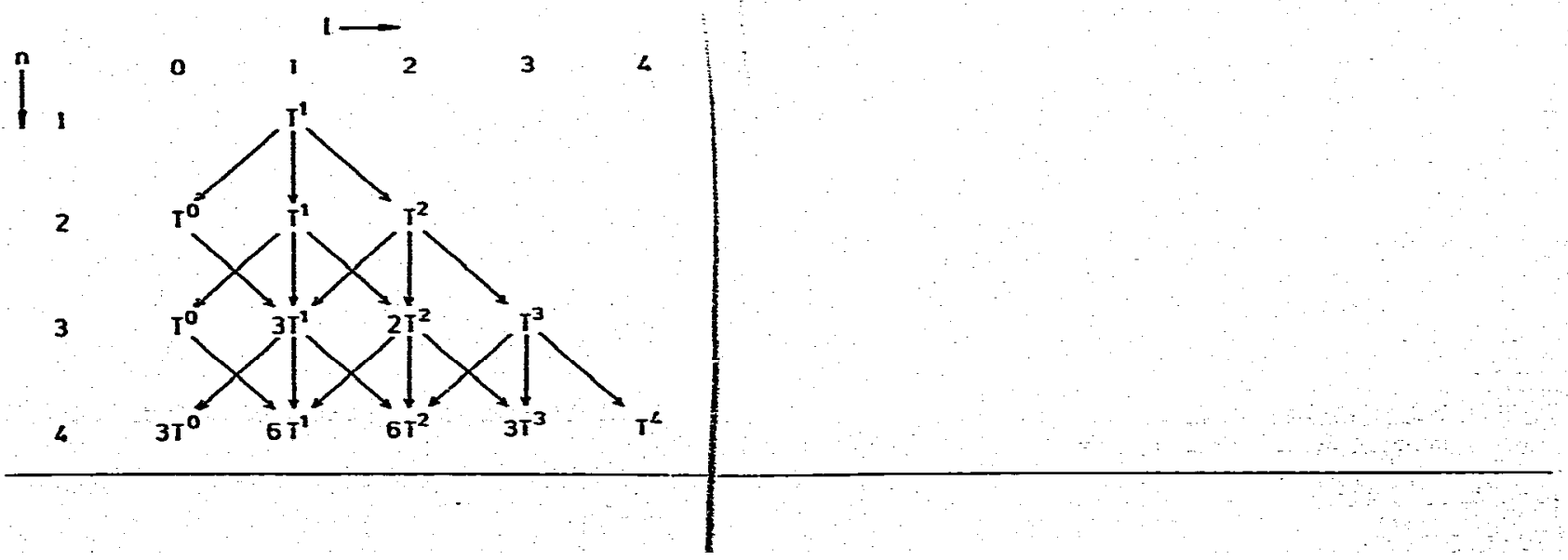


An alternative basis system to express the components of $x^{(2)}$ is given by the irreducible tensor operators $T_{m}^{l}$ of the rotation group [57]. Under rotation of the coordinate system the $2 l+1$ components of $T^{l}$ transform like the spherical harmonics Table 1 shows in a diagram how the cartesian tensors of increasing rank can be decomposed into irreducible tensors $T^{\prime}$. The tensors of the next higher rank are found by multiplication of each $T^{\prime}$ of te lower-rank tensor with $T^{l}$ followed by reduction For $I \geqslant 1$ this yields three new tensors in each step according to:

$$
T^{i} \otimes T^{1}=T^{\ell-1}+T^{l}+T^{t-1}
$$

The third-rank tensor $x^{(2)}$ decomposes into one $T^{0}$, three $T^{1}$, two $T^{2}$, and one $T^{3}$. Tensor operators with the same $I$ must be distinguished by a further index $q$ which we take to be the $l$ of the parent irreducible tensor operator of rank $n=2$

Under rotation of the physical system with angle $\varphi$ around the $Z$ axis - the axis of quantization of the angular momentum - the irreducible tensor operators transform in the following way:

$$
T_{m}^{l}(\varphi)=T_{m}^{l}(0) \exp (\mathrm{im} \varphi)
$$

The components with $m=0$ are the invariants. According to table 1 seven such invariants exist in the case of $x^{(2)}$ in complete agreement with the argument in cartesian coordinates. Transformation between both basis systems is accomplished by a unitary matrix $c$ relating the cartesian basis tensors $|i j k\rangle$ and the irreducible basis tensors $\mid$ lqm $\rangle$ :

$$
|l q m\rangle=\sum_{i j k} c_{I q m, i j k}|i j k\rangle
$$

The coupling scheme adopted here first couples the two cartesian indices $j k$ corresponding to the field

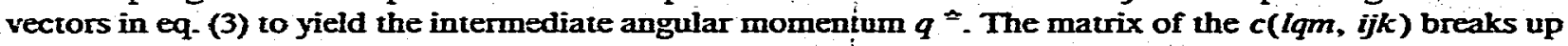
into two blocks which are given in table 2 . Writing the components of $x$ defined in both tensor bases as

$$
x_{i j k}=\langle x \mid \bar{j} j k\rangle, \quad x_{m}^{\prime \cdot q}=\langle x \mid l q m\rangle,
$$

this matrix serves to interconvert $X$ in toth systems:

$$
x_{m}^{l-q}=\sum_{i j k} c_{l q m, i j k} X_{i j k}, \quad X_{i j k}=\sum_{l q m} c_{l q m, i j k}^{*} X_{m}^{\prime-q}
$$

whereas the matrix $c(I q m, i j k)$ is unitary, the matrix relating the rotational invariants in both systems is not $=$ The reason for this is that columns corresponfing to $x_{z x x}$ and $x_{z Y r}$ etc. have been contracted. The result is:

$$
\begin{aligned}
& \left(\begin{array}{l}
x_{0}^{1.0} \\
x_{0}^{1.1} \\
x_{0}^{1.2} \\
x_{0}^{32}
\end{array}\right)=\left(\begin{array}{cccc}
-1 / \sqrt{3} & -2 / \sqrt{3} & 0 & 0 \\
0 & 0 & -1 & 1 \\
-2 / \sqrt{15} & 2 / \sqrt{15} & -\sqrt{3 / 5} & -\sqrt{3 / 5} \\
\sqrt{2 / 5} & -\sqrt{2 / 5} & -\sqrt{2 / 5} & -\sqrt{2 / 5}
\end{array}\right)\left(\begin{array}{l}
x_{z Z Z} \\
x_{z x x} \\
x_{x z x} \\
x_{x x z}
\end{array}\right) \text {, } \\
& \left(\begin{array}{l}
x_{0}^{0 . x} \\
x_{0}^{2.1} \\
x_{0}^{\frac{1.2}{3}}
\end{array}\right)=i\left(\begin{array}{ccc}
\sqrt{2 / 3} & \sqrt{2 / 3} & \sqrt{2 / 3} \\
1 / \sqrt{3} & 1 / \sqrt{3} & -2 / \sqrt{3} \\
-1 & 1 & 0
\end{array}\right)\left(\begin{array}{l}
x_{x y z} \\
x_{y z x} \\
x_{z x Y}
\end{array}\right) \text {, }
\end{aligned}
$$

- Maker [55] used a similar unitary transformation for third-rank trusors in his treatment of non-linear light scattering However. both his coupling scheme and phase conventions are different from ouss.

1 This is the reason why we defined the coefficients $c(\operatorname{lom} i j k)$ as relating orthogonal basis tensors and not $x$-components. 
and the inverse:

$$
\begin{aligned}
& \left(\begin{array}{l}
x_{z z z} \\
x_{z x x} \\
x_{x z x} \\
x_{x x z}
\end{array}\right)=\left(\begin{array}{cccc}
-1 / \sqrt{3} & 0 & -2 / \sqrt{15} & \sqrt{2 / 5} \\
-1 / \sqrt{3} & 0 & 1 / \sqrt{15} & -1 / \sqrt{10} \\
0 & -1 / 2 & -\sqrt{3 / 20} & -1 / \sqrt{10} \\
0 & 1 / 2 & -\sqrt{3 / 20} & -1 / \sqrt{10}
\end{array}\right)\left(\begin{array}{l}
x_{0}^{1.0} \\
x_{0}^{1.1} \\
x_{0}^{1.2} \\
x_{0}^{3.2}
\end{array}\right) \\
& \left(\begin{array}{l}
x_{x y z} \\
x_{Y z x} \\
x_{z x Y}
\end{array}\right)=-1\left(\begin{array}{ccc}
1 / \sqrt{6} & 1 / \sqrt{12} & -1 / 2 \\
1 / \sqrt{6} & 1 / \sqrt{12} & 1 / 2 \\
1 / \sqrt{6} & -1 / \sqrt{3} & 0
\end{array}\right)\left(\begin{array}{l}
x_{0}^{0.1} \\
x_{0}^{2.1} \\
x_{0}^{2.2}
\end{array}\right)
\end{aligned}
$$

The reducticn into irreducible tensor operators can also be done with the molecular tensor in the molecule-fixed coordinate system. The unitary transformation. matrix $c(l q m, i j k)$ is exactly the same, and eqs. (12) apply with $\chi$ replaced by $\beta$.

\section{The orientation distribution function}

\section{I. The orientafion acerage}

We assume that the macroscopic susceptibility $x$ is the tenscr sum of the molecular hyperpolarizabilities $\beta$, therewith neglecting contributions which might anise from the mutual interaction of the molecules:

$$
\mathrm{x}=\langle\beta\rangle=\int \mathrm{d} \Omega f(\Omega) \beta(\Omega)
$$

For ensembles of organic molecules this approximation is usually correct, especially in dilute solutions. The average is defined by the orientation distribution function $f(\Omega)$ giving the probability that a molecule is found with onientation $\Omega$ of its molecular coordinate system with respect to the laboratory coordinate system. $\Omega$ denotes a set of three orientational parameters, in particular the Euler angles $\alpha, \beta, \gamma$ as shown in fig. 1. The rotation between the reference system $x y z$ attached to the molecule and the laboratory system $X Y Z$ can be viewed as accomplished in two ways: Consecutive rotation with angles $\alpha, \beta, \gamma$ around the axes $z, y, z$ moving with the molecule, or consecutive rotations with the same angles but in opposite order $\gamma, \beta$, $\alpha$ around the laboratory-fixed axes $Z, Y, Z$. In eq. $(15) \beta(\Omega)$ is the hyperpolarizability contribution of a molecule with orientation $\Omega$ expressed in the laboratory coordinates. It is found from the tensor in the molecular coordinates $\beta(\Omega=0)$ by a rotation operator. In cartesian coordinates:

$$
\beta_{I J K}(\Omega)=\sum_{i j k} R_{I i}(\Omega) R_{J j}(\Omega) R_{K k}(\Omega) \beta_{i j k}
$$

where $R$ is the matrix of the direction cosines relating the two reference frames. At this point the simplicity of the irreducible tensor operator formalism comes in with the much simpler expression:

$$
\beta_{n}^{\prime}(\Omega)=\sum_{m=-1}^{l} D_{m n}^{\prime}(\Omega) \beta_{m}^{l}(0)
$$

With the above given definition of Euler angles and rotations the Wigner matrices $D_{m n}^{\prime}$ are given by:

$$
D_{m i n}^{\prime}(\Omega)=\exp [\mathrm{i}(m \gamma+n \alpha)] d_{m n}^{l}(\beta)
$$




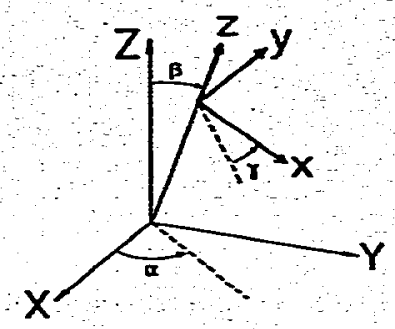

\section{$\mathrm{S} \times z$}
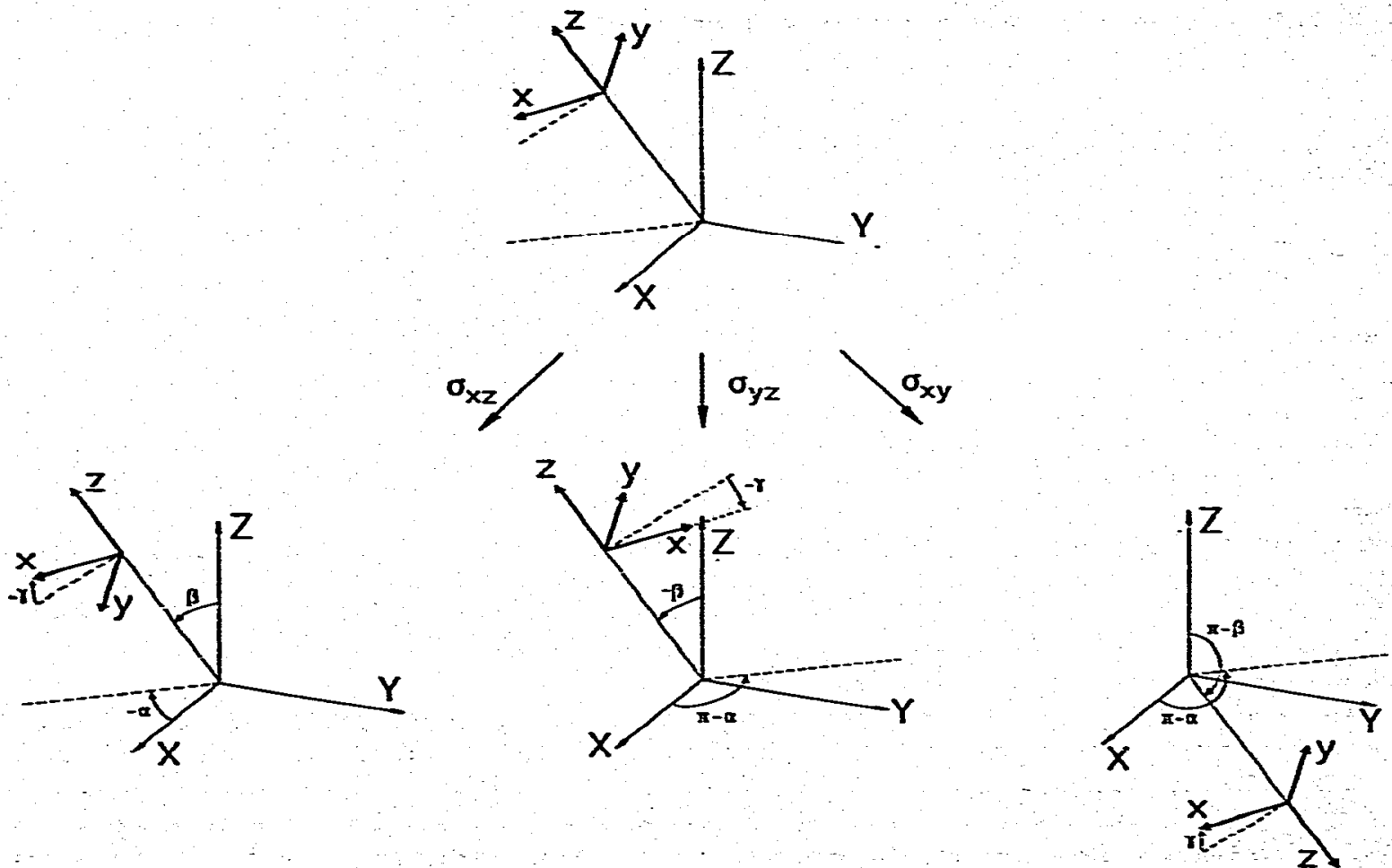

Fig 1. Relative orientation of laboratory frame $X Y Z$ and molecule fixed frame $x y z$ described by Euler angles $\alpha, \beta, \gamma$. The symmetry conditions for the orientation distribution function are found by first applying the macrascopic mirror opezanion and subsecuenty the molecular mintor operation $\sigma_{x z}, \sigma_{y z}$ or $\sigma_{x y}$ Soe text for discussion.

and $d_{m i}^{l}(\beta)$ is a real function discussed in several standard texts on angular momentum [57-59]-In our notation the rotation about the figure axis of the system $(\gamma)$ is associated with the first index $(m)$ in $D_{\min }^{\prime}$ This follows the recommendations of Wolf [60] and Silver [57]. The Wigner matrices form a complete set of functions in the space $(\alpha, \beta, \gamma)$, and the distribution function can be expanded:

$$
f(\Omega)=\sum_{m=n}(2 l+1) B_{m n}^{*} D_{m n}^{l}(\Omega)
$$


with the expansion coefficients:

$$
B_{l m n}=\left\langle D_{m n}\right\rangle \text {. }
$$

The average of eq. (17) yields:

$$
\chi_{n}^{l}=\left\langle\beta_{n}^{r}\right\rangle=\sum_{m} B_{l m n} \beta_{m}^{l}(0)
$$

The $2 l+1$ components of each irreducible tensor average only among themselves and not with those of another $l$, or the same $l$ but another subindex $q$. Consequently the tensors with $l=0$ remain unchanged by any average and are called isotropic tensors. The coefficient $B_{000}$ is always unity since eq. (20) is just the normalization requirement for $f(\Omega)$ in this case. In isotropic systems $B_{000}$ is the only non-vanishing coefficient and the $x_{0}^{0}$ are the isotropic invariants of $\chi$. It is seen from table 1 that one isotropic invariant exists for the third-rank tensor, and three for the fourth-rank tensor. The latter correspond to the three parameters measurable in two-photon absorbtion in isotropic media [61-63]-

\subsection{Symmetry}

So far we have not imposed any symmetry constraint upon eq. (20). The postulate of rotational invariance allows only the components $\chi_{m}^{t . q}$ with $m=0$, as shown in section 3.1:

$$
X_{0}^{l-q}=\sum_{m=-l}^{T} \beta_{m}^{t-q} B_{l m 0} .
$$

The property of the Wigner matrices $D_{m n}^{\prime *}=(-1)^{n x-n} D_{-m-n}^{2}$ gives a further condition:

$$
B_{l m 0}=(-1)^{m} B_{l-m 0}^{*} \text {. }
$$

which ensures that the distribution function is real. Therefore a maximum of 15 real parameters describe the distribution function up to $I=3$. This number is further reduced when additional symmetry elements are present.

Let us assume that the distribution also contains symmetry planes containing the $Z$ axis. Due to the rotational symmetry only one plane needs to be considered, say $S_{x z}$. Since molecules with no mirror plane cannot be arranged in such a way as to give the whole ensemble mirror symmetry, a mirror plane must exist in the molecule as well. Fig. 1 shows how the consequences for the distribution function are found. First, a macroscopic mirror plane transforms an orientational site $(\alpha, \beta, \gamma)$ into an equally probable one. In the second step the molecular symmetry plane is used to make the molecular frame right handed again. Finally, the set of Euler angles $\left(\alpha^{\prime}, \beta^{\prime}, \gamma\right)$ is found that would have produced this orientation purely by rotation. With $\sigma_{x=}$ being the molecular mirror plane we find $f(\alpha, \beta, \gamma)=f(-\alpha, \beta,-\gamma)$ leading to $B_{I m n}=B_{t m n-}^{*}$ If the molecular symmetry plane is $\sigma_{y=}$, the condition is $f(\alpha, \beta, \gamma)=f(=-\alpha,-\beta,-\gamma)$ resulting in $B_{l m n}=$ $B^{*}{ }_{l m n}(-1)^{m}$. Finally, for the $\sigma_{x y}$ plane $f(\alpha, \beta, \gamma)=f(\pi-\alpha,=-\beta, \gamma)$ and $B_{I m n}=(-1)^{l-m+n} B_{I m-n^{-}}$All three cases are of course equivalent, since they only differ in the choice of the molecule-fixed axis system. In combination with the result for the rotational invariance the number of real parameters up to $l=3$ is reduced to 9 with one mirror plane. Combining the results for a molecule with two mirror planes $\sigma_{x=}$ and $\sigma_{y z}$ only 5 parameters are left. With all three molecular mirror planes this number reduces to 2 , namely $B_{200}$ and $B_{220}$ both being real.

In a similar way other symmetry constraints can be imposed. Eg, if the molecules were themselves rotationally symmetric around their $z$ axes, the distribution function fould become independent of $\gamma$, and the parameters

$$
B_{r 00}=\left\langle P_{I}(\cos \beta)\right\rangle
$$


are the only non-vanishing expansion coefficients.

With this formalism we are now in the position to connect the microscopic quantities with the macroscopic ones. We will present two examples, one starting with the knowledge of the distribution function, the other with the knowledge of the dominant molecular tensor elements.

\section{Determination of molecular tensor components in a system with known orientational distribution}

The general distribution function for a rotationally symmetric ensemble depends on the two orientational coordinates $\beta$ and $\gamma$. In many cases, however, all angles $\gamma$ are equally probable and the distribution function is only dependent on the angle $\beta$ giving the inclination of the molecular figure axis $z$ to the polar axis of the sample. This can be due to molecular symmetry, e.g. when the molecule can be considered as cylindrically symmetric. It can also be due to the mechanism of the orientation. One example is the orientation of permanent dipoles in a static electric field, leading to the distribution:

$$
\begin{aligned}
& f(\beta)=\exp (-x \cos \beta)\left[\int \mathrm{d} \Omega \exp (-x \cos \beta)\right]^{-1} \\
& x=\mu E / k T \text {. }
\end{aligned}
$$

Since $f$ does not depend on $\alpha$ and $\gamma$, the only non-vanishing expansion coefficients $B_{l m n}$ are those with $m=n=0$. For the oriented dipole distribution function these are:

$$
\begin{aligned}
& B_{100}=1 / x-\operatorname{coth}(x), \quad B_{200}=1+3 / x^{2}-(3 / x) \operatorname{coth}(x), \\
& B_{300}=6 / x+15 / x^{3}-\left(1 \div 15 / x^{2}\right) \operatorname{coth}(x)
\end{aligned}
$$

For small parameters $x$ they can be expanded:

$$
B_{100} \propto-\frac{1}{3} x+\frac{1}{43} x^{3}-\frac{2}{33} x^{5}, \quad B_{200} \propto \frac{1}{15} x^{2}-\frac{1}{315} x^{4}, \quad B_{300} \propto-\frac{1}{105} x^{3}+\frac{1}{23} x^{5}:
$$

The following discussion is, however, independent of the particular form of the $B_{100}$ coefficients. Performing the average of $\beta$ according to eq. (22) gives the simple result:

$$
X_{0}^{l \cdot q}=B_{100} \beta_{0}^{l-q}
$$

The seven experimentally accessible parameters $\chi_{0}^{l \cdot 9}$ correspond directly to seven molecular parameters $\beta_{0}^{l . q}$. These can also be expressed as linear combinations of the $\beta_{i j k}$ elements in cartesian coordinates via the coefficients given in table 2 . Thirteen $\beta_{i j k}$ components are involved, but only seven independent linear combinations appear. They can be written in matrix form as:

$$
\begin{aligned}
& \left(\begin{array}{l}
\beta_{0}^{1.0} \\
\beta_{0}^{1.1} \\
\beta_{0}^{1.2} \\
\beta_{0}^{32}
\end{array}\right)=\left(\begin{array}{cccc}
-1 / \sqrt{3} & -1 / \sqrt{3} & 0 & 0 \\
0 & 0 & -1 / 2 & 1 / 2 \\
-2 / \sqrt{15} & 1 / \sqrt{15} & -\sqrt{3 / 20} & -\sqrt{3 / 20} \\
2 / \sqrt{10} & -1 / \sqrt{10} & -1 / \sqrt{10} & -1 / \sqrt{10}
\end{array}\right)\left(\begin{array}{l}
\beta_{z=z} \\
\beta_{z x x}+\beta_{z y y} \\
\beta_{x x x}+\beta_{y=y} \\
\beta_{x x=}+\beta_{y y=}
\end{array}\right) \\
& \left(\begin{array}{l}
\beta_{0}^{0.1} \\
\beta_{0}^{2.1} \\
\beta_{0}^{2.2}
\end{array}\right)=1\left(\begin{array}{ccc}
1 / \sqrt{6} & 1 / \sqrt{6} & 1 / \sqrt{6} \\
1 / \sqrt{12} & 1 / \sqrt{12} & -1 / \sqrt{3} \\
-1 / 2 & 1 / 2 & 0
\end{array}\right)\left(\begin{array}{l}
\beta_{x y=}-\beta_{y x=} \\
\beta_{y=x}-\beta_{x=y} \\
\beta_{x x y}-\beta_{y x x}
\end{array}\right)
\end{aligned}
$$

These matrices are the transposed of those in eq. (14). Therefore, the above given equations can be solved 
for the cartesian components with the inverted matrices given as the transposed of the matrices in eq. (13). By successive multiplication of eqs. (30), (29), and (14) we can express the cartesian components of $x$ in those of $\beta$ :

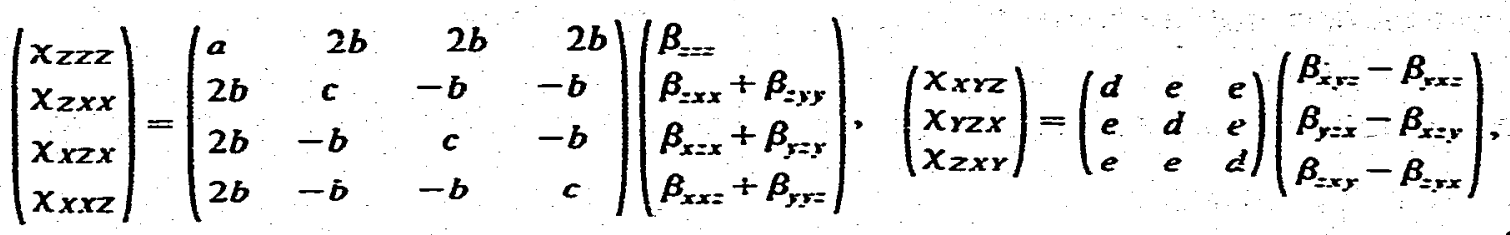

with

$$
\begin{array}{ll}
a=\left(3 B_{100}+2 B_{300}\right) / 5, & b=\left(B_{100}-B_{300}\right) / 10, \\
c=\left(4 B_{100}+B_{300}\right) / 10, & d=\left(1+2 B_{200}\right) / 6, \quad e=\left(1-B_{200}\right) / 6
\end{array}
$$

and the inverted problem is:

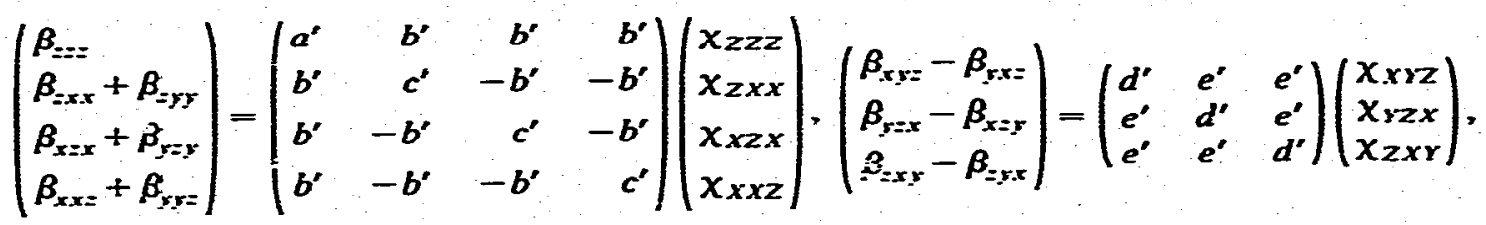

with

$$
\begin{aligned}
& a^{\prime}=\left(3 / E_{100}+2 / B_{300}\right) / 5, \quad b^{\prime}=2\left(1 / B_{100}-1 / B_{300}\right) / 5, \\
& c^{\prime}=2\left(4 / B_{100} \div 1 / B_{300}\right) / 5, \quad d^{\prime}=2\left(1+2 / B_{200}\right) / 3, \quad e^{\prime}=2\left(1-1 / B_{200}\right) / 3 .
\end{aligned}
$$

It should be noted that this inversion and consequently the determination of all seven molecular tensor comporents is only possible when the $B_{i 00}$ are sufficiently large. From $B_{200} \ll 1$ follows $g=h=1 / 6$ in eq. (31) with the consequence:

$$
x_{X Y Z}=x_{r Z X}=X_{Z X Y}
$$

while with $B_{300} \ll B_{100}$ we have:

$$
x_{z z z}=x_{z x x} \div x_{x z x}+x_{x x z}
$$

and this linear dependence prevents inversion of the matrices in eq. (31). Only two molecular parameters are accessible in these cases, namely:

$$
\begin{aligned}
& \bar{\beta}_{1}=X_{x y z}=\frac{1}{6}\left(\beta_{x y z}-\beta_{y x=}+\beta_{y=x}-\beta_{x=y}+\beta_{z x y}-\beta_{z y x}\right), \\
& \bar{\beta}_{2}=X_{z z z} / B_{100}=\frac{1}{5} \sum_{i=1}^{3}\left(\beta_{z i i}+\beta_{i=i}+\beta_{i i z}\right) .
\end{aligned}
$$

The first term is the isotropic term which vanishes for non-chiral molecules. The second parameter is the one measured in conventional field-induced second-harmonic generation [25-27,43].

With molecules having large dipole moments in the electronic ground state one can hope to archive considerable alignment using field strengths below the threshold of breakthrough [53,54]. In fact, saturation of the fiela-induced second-harmonic generation has been observed [31]. Since highly polar molecules are also very likely to have large hyperpolarizabilities, the above outlined procedure seems a promising method. 
Often molecular symmetry will reduce the number of non-vanishing tensor components considerably, and the left-hand side of eq. (32) is simplified accordingly. The frequently encountered case of planar s-electron systems is discussed in section 5 .

\section{Determination of orientational parameters when the molecular tensor is known}

Up to 15 orientational parameters can be involved in the average, but only seven quantities can be measured. The number of independent parameters is reduced by symmetry properties of the sample or the molecules as discussed in sections 2 and 3 . For a molecule with $C_{2 v}$ symmetry only seven tensor elements do not vanish by symmetry. With $z$ as the twofold axis these are $\beta_{z=z}, \beta_{z x x}, \beta_{z y y}, \beta_{x x z=}, \beta_{x=x}, \beta_{y y z}$, and $\beta_{y=y}$. If in addition the molecule is a planar $\pi$-electron system, the $\beta$-components containing the out of plane coordinate index involve two transition moments to $\mathrm{nr} *$ excited states in the perturbative expression for $\beta$ [64]. Semi-empirical calculations for aniline and $p$-nitroaniline indicate that these tensor components are indeed very small for visible and near UV light [64]: The remaining components are $\beta_{z=-}, \beta_{z x x}, \beta_{x=x}$ and $\beta_{x x=-}$ When no mirror plane perpendicular to the molecular plane exists, the additional tensor components $\beta_{x=3}, \beta_{z x=}$ and $\beta_{z=x}$ have to be considered. Table 3 shows how these enter into the macroscopic susceptibility. The results have been obtained by first expressing the $\beta_{m}^{\prime}$ in terms of the $\beta_{i j k \text { - In the second }}$ step the average was performed according to eq- (22) and using the symmetry properties of the $B$ coefficients for a molecule with $x z$ mirror plane. For example, $\beta_{z z}$ yields the contributions:

$$
\beta_{0}^{1.0}=-\beta_{z=2} / \sqrt{3}, \quad \beta_{0}^{12}=-2 \beta_{z z=} / \sqrt{15}, \quad \beta_{0}^{32}=\sqrt{2 / 5} \beta_{z=:}
$$

leading to:

$$
x_{0}^{1.0}=-B_{100} \beta_{z=:} / \sqrt{3}, \quad x_{0}^{1.2}=0, \quad x_{0}^{1.2}=-2 B_{100} \beta_{z: z} / \sqrt{15}, \quad x_{0}^{3.2}=\sqrt{2 / 5} B_{300} \beta_{z=:},
$$

giving the first column of the matrix in table 3 . Table 4 gives the result for the macroscopic tensor in the cartesian coordinate representation.

With the above given symmetry restrictions five out of the possible nine orientational parameters are involved in the averaged values accessible by sum- and difference-frequency mixing experiments. The assumption of a planar chromophore with negligible contributions from out-of-plane transition moments causes all $\chi_{0}^{l}$ with $l$ even to vanish. Table 3 shows that the three $\chi_{0}^{1}$ contain only two orientational

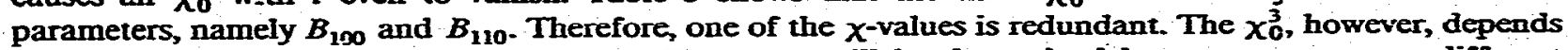
on three parameters $B_{300} B_{310}$, and $B_{320}$. These can still be determined by measurements at different frequencies chosen in such a way that they single out particular matrix elements of $\beta$ through resonance with accordingly polarized transitions. When the three frequencies $\omega_{1}, \omega_{2}$, and $\omega_{3}=\omega_{2} \pm \omega_{1}$ are in

Table 3

Contribution of various molecular tensor components $\beta_{i j k}$ to the macroscopic invariants $x_{0}^{\prime-q}$

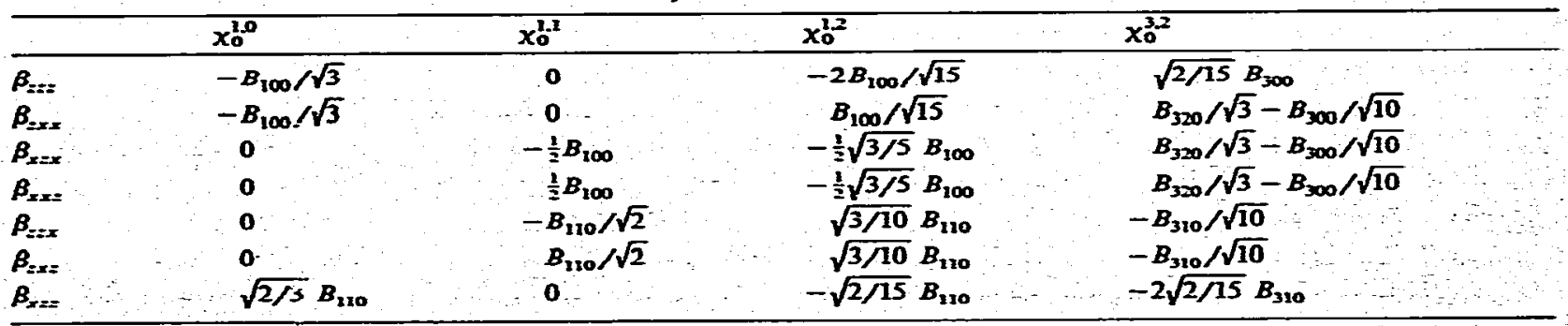



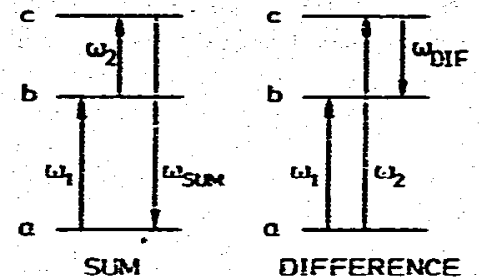

Fig 2 Definition of level schemes for fully resonant sum- and difference-frequency generation.

resonance with a molecular three level system $(a, b, c)$ as shown in fig. 2 , the dominant resonant part of $\beta$ will be prcportionai to the tensor product of the corresponding transition dipoles With states $a, b, c$ having symmetry $A_{1}, B_{2}$, and $A_{1}$ of point group $C_{2 v}$, for example, the dominant tensor elements are $\beta_{\text {exx }}$ (sum) and $\beta_{x x=}$ (dif), while with all states having symmetry $A_{1}$ it will be $\beta_{z=z}$ for both processes: With $\beta_{z=z}$ dominant the measurement will yield $B_{300}$ - With other frequencies involving $\beta_{\text {.xx }}$ as the dominant tensor component the linear combination $B_{300}-(10 / 3)^{1 / 2} B_{320}$ is measured, while with $\beta_{x=2}$ dominant one gets $B_{310}-$

Let us consider some cases of interest:

(i) $\beta_{z=z}$ is dominant. (This is the special case treated in ref. [45]). From table 4 we obtain:

$$
x_{z x x}=\lambda_{x z x}=x_{x x z}
$$

The orientational parameter ratio is:

$$
B_{300} / B_{100}=\left(x_{z z z}-3 x_{z x x}\right) /\left(x_{z z z}+2 x_{z x x}\right)
$$

leading to

$$
\left\langle\cos ^{3} \beta\right\rangle /\langle\cos \beta\rangle=x_{z z z} /\left(x_{z z z}+2 x_{z x x}\right) \text {. }
$$

Ratios of this kind are easier to obtain than absolute values of $\chi$. For sufficiently sharp distributions of the orientation angle this ratio can be interpreted as $\cos ^{2} \beta_{0}$ and the average angle $\beta_{0}$ can be extracted.

(ii) $\beta_{=x x}$ is dominant. Table 4 now leads to:

$$
x_{z z z}=-2 x_{x z x}=-2 x_{x x z}
$$

and

$$
\left(B_{300}-B_{320} \sqrt{10 / 3}\right) / B_{100}=\left(2 x z x x-4 x_{z z z}\right) /\left(x_{z z z}+2 x z x x\right)
$$

Table 4

Results of table 4 transformed to invarients $x_{i j k}$ in cartesian coordinates

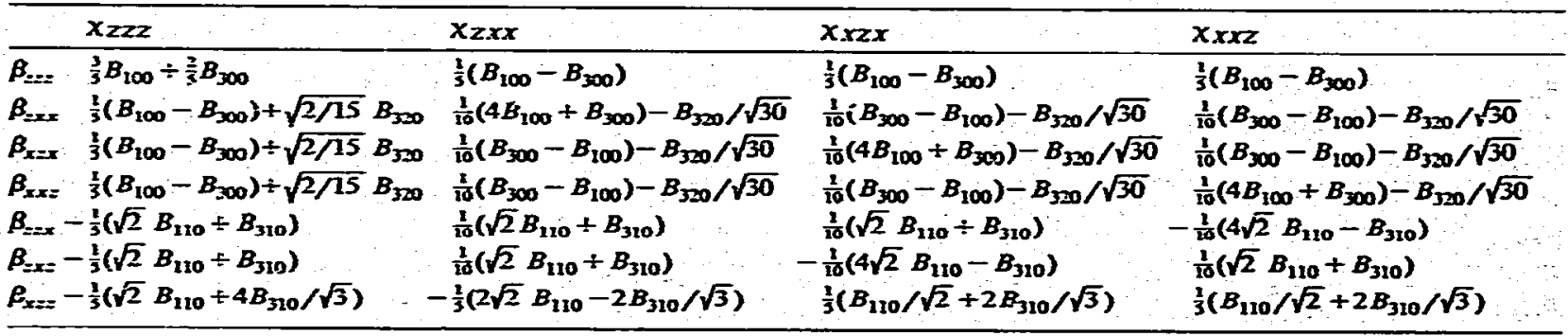


This can be solved for $B_{320}$ if $B_{300}$ is already known $B_{320}$ depends on the distribution of the angle $\gamma$ around the figure axis of the molecule:

$$
B_{320}=(15 / \sqrt{5 !})\left\langle\mathrm{e}^{-2 i \gamma}\left(\cos \beta-\cos ^{3} \beta\right)\right\rangle
$$

It vanishes if $\boldsymbol{\gamma}$ is randomly distributed. When the distribution of $\beta$ and $\gamma$ are independent it follows:

$$
\sqrt{10 / 3} B_{320}=\left(B_{100}-B_{300}\right)\left\langle\mathrm{e}^{-2 i r}\right\rangle \text {. }
$$

For a random distribution of $\gamma$ we get:

$$
\left\langle\cos ^{3} \beta\right\rangle /\langle\cos \beta\rangle=\left(2 x_{z x x}-x_{z z z}\right) /\left(2 x z x x+x_{z z z}\right) \text {, }
$$

whereas the other extreme case $\gamma=0$ rogether with eq. (44) yields:

$$
\left\langle\cos ^{3} \beta\right\rangle /\langle\cos \beta\rangle=2 x_{z x x} /\left(x_{z z z}+2 x_{z x x}\right) \text {. }
$$

This corresponds to the orientation model adopted in ref. [44] for rhodamine- $6 \mathrm{~g}$ on fused silica surfaces.

(iii) $\beta_{x x=}=\beta_{x=x}$ is dominant. This leads to:

$$
x_{z z z}=-2 x_{z x x}, \quad x_{x z x}=x_{x x z}
$$

and

$$
\left(B_{300}-B_{330} \sqrt{10 / 3}\right) / B_{100}=\left(3 x_{2 x X}+2 x_{x x Z}\right) /\left(2 x_{x x z}-2 x_{z X X}\right) \text {, }
$$

which for random $\gamma$ gives:

$$
\left\langle\cos ^{3} \beta\right\rangle /\langle\cos \beta\rangle=\chi_{x x z} /\left(\chi_{x x z}-\chi_{z x x}\right) \text {. }
$$

while for $\gamma=0$ it gives:

$$
\left\langle\cos ^{3} \beta\right\rangle /\langle\cos \beta\rangle=\left(2 x_{x x z}-x_{z x x}\right) /\left(2 x_{x x z}-2 x_{z x x}\right)
$$

(iv) $\beta_{=:=}$and $\beta_{=x x}$ both give a dominant contribution. This could arise in sum-frequency experiments where the outgoing frequency is resonant with a $z$-polarized transition while no real intermediate state is resonant with the ingoing beams. In this case is:

$$
\begin{aligned}
& x_{x z x}=x_{x x z} \\
& \beta_{z z=} / \beta_{z x x}=\left(x_{z z z}+2 x_{x x z}\right) /\left(2 x_{z x x}-2 x_{x x z}\right)
\end{aligned}
$$

and the two extremal values for the orientational ratio are for random $\gamma$ :

$$
\left\langle\cos ^{3} \beta\right\rangle /\langle\cos \beta)=\left(x_{z z z}-x_{z x x}+x_{x x z}\right) /\left(x_{z z z}-x_{z x x}+3 x_{x x z}\right)
$$

and for $\boldsymbol{\gamma}=\mathbf{0}$ :

$$
\left\langle\cos ^{3} \beta\right\rangle /\langle\cos \beta\rangle=\left(x_{z z z}-2 x z x x+2 x x x z\right) /\left(x_{z z z}-2 x z x x+4 x x x z\right)
$$

We can now attempt an interpretation of the recently determined relative values $x z z z=0.692, x z x x=1.0$, $x_{X Z X}=x_{x x z}=-0.158$ for thodamine $-6 \mathrm{~g}$ adsorbed on fused silica [52]. The molecule has almost $C_{2 \mathrm{v}}$ symmetry and the generated second-harmonic light is resonant with a $z$-polarized transition. Taking the symmetry $B_{2}$ of the $S_{1}$ state for the intermediate state, $\beta_{2 x x}$ is expected to be dominant However, eq. (41) is not fulfilled, although the sign is correct. This suggests that some contribution from $\beta$ is present as discussed in case (iv) above. Eq. (52) gives:

$$
\beta_{z=2} / \beta_{z x x}=0.16 \text {, }
$$


which sounds reasonable. For the orientational parameter follows:

$$
\begin{aligned}
\left\langle\cos ^{3} \beta\right\rangle /\langle\cos \beta\rangle & =0.5959 \quad \gamma \text { random, } \\
& =0.8371 \quad \gamma=0,
\end{aligned}
$$

which, for a sharp distribution would indicate a mean angle $\beta_{0}$ of:

$$
\begin{aligned}
\beta_{0} & =39.5^{\circ} & \gamma \text { random } \\
& =23.8^{\circ} & \gamma=0 .
\end{aligned}
$$

It seems that the more the angle $\gamma$ is restricted the more will $\beta_{0}$ tend to smaller angles. With $\gamma=0$ the molecular $y$ axis is restricted to lie in the surface plane, and $\beta_{0}=24^{\circ}$ will place the molecular plane at an angle of $66^{\circ}$ to the surface. It seens more reasonable to assume more freedom in the orientational parameter and consequently allow the molecular dipole moment to come closer to the surface. However, the present data allow no further conclusions. Hopefully future experiments with frequencies favouring $\beta$ z=: wili resolve this ambiguity.

\section{Determination of macroscopic $x^{(2)}$ tensor elements}

The generated wave at the sum or difference frequency is found as the solution of an inhomogeneous wave equation for the non-linear medium:

$$
\nabla \times \nabla \times \mathscr{E}+\left(n^{2} / c^{2}\right) \bar{E}=-\left(4 \pi / c^{2}\right) \dot{9} .
$$

Here $n$ is the refractive index for the generated wave, and $\mathcal{P}$ is the so-called source wave given by eq- (3). Outside the non-linear medium all waves propagate as free waves, which are found through application of the various boundary conditions. A realistic and still not too complicated case is given by the parallel non-linear slab as scetched in fig. 3.

The two ingoing beams $E_{1}, E_{2}$ enter the furst boundary with angles of incidence $\vartheta_{1}, \vartheta_{2}$ and are refracted into the noz-linear medium. Here they travel under the angles $\vartheta_{1}^{\prime}, \vartheta_{2}^{\prime}$ with amplitude vectors $E_{1}^{\prime}, E_{2}^{\prime}$ given by Fresnels equations. These in turn form the source wave:

$$
\mathcal{G}(r, t)=\mathrm{x}^{(2)} \cdot E_{1} E_{2} \exp \left[\mathrm{i}\left(k_{\mathrm{s}}-r-\omega_{3} t\right)\right], \quad \omega_{3}=\omega_{1}+\omega_{2}, \quad k_{\mathrm{s}}=k_{1}+k_{2-}
$$

The source wave has an effective refractive index $n_{s}=k_{s} c / \omega_{3}$. As Bloembergen and Pershan have shown [65], all non-linear waves will travel in the plane spanned by $k_{s}$ and the surface normal $z^{\prime}$; which is called the plane of incidence and taken to be the $x^{\prime} z^{\prime}$ plane in fig. 3 .

The non-linear medium is in general birefringent with refractive indices $n_{\mathrm{a}}$ and $\boldsymbol{n}_{\perp}$ for beams polarized parallel or perpendicular to the optical axis. The latter is identical with the orientation axis $z$ around which the sample is rotationally invariant. In order to avoid spliting of the beams into ordinary and extraordinary beams we reake the further assumption that the orientation axis and the wave vectors of the ingoing beams also lie in the plane of incidence In praxis the orientation axis will often coincide with the surface normal, e.g in surface studies or in thin samples oriented by an electric de field. In this case $x^{\prime}=x, z^{\prime}=z$, and $\varphi=\vartheta_{S}$. To ensure constant interaction of the beams inside the non-linear medium one could further choose $\vartheta_{1}$ and $\vartheta_{2}$ so that after refraction $\vartheta_{1}=\vartheta_{2}$, but this is not necessary for the following formalism.

Considering only s- or p-polarized incident waves we have inside the non-linear medium:

$$
E_{I}^{\prime}=\frac{2 n_{1} \cos \vartheta_{1}}{n_{\perp} \cos \vartheta_{1}+n_{\perp}^{\prime} \cos \vartheta_{S}} E_{\perp}, \quad E_{\mathrm{a}}^{\prime}=\frac{2 n_{\mathrm{a}} \cos \vartheta_{1}}{n_{\mathrm{I}} \cos \vartheta_{\mathrm{S}}+n_{\mathrm{I}}^{\prime} \cos \vartheta_{1}} E_{\mathrm{I}}
$$




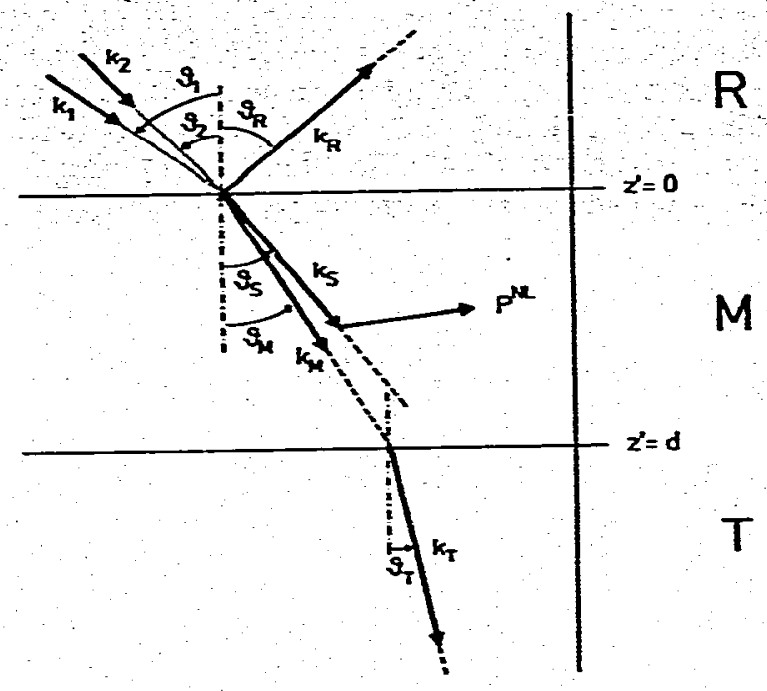

Fig. 3. Wave vectors and poiarization directions for sum- and difference-frequency generation in a parallel non-linear slab. For discussion see text.

With both beams crossing the orientation axis under the same angle $\varphi$ the following non-linear polarizations are generated:

$$
\begin{aligned}
& E_{1}^{\prime} E_{21}^{\prime}= \\
& P_{X}=0, \quad P_{Y}=0, \quad P_{Z}=x_{Z X X} \\
& E_{10}^{\prime} E_{2:}^{\prime}: \\
& P_{X}=\left(X_{X Z X}+\chi_{X X Z}\right) \sin \varphi \cos \varphi, \quad P_{Y}=-\left(X_{X Z Y}+X_{X Y Z}\right) \sin \varphi \cos \varphi \text {, } \\
& P_{Z}=X z x x \cos ^{2} \Phi+x z z z \sin ^{2} \varphi ; \\
& E_{1:}^{\prime} E_{21}^{\prime}= \\
& P_{X}=\chi_{X Z Y} \sin \varphi, \quad P_{Y}=\chi_{X Z X} \sin \varphi, \quad P_{Z}=-\chi_{Z X Y} \cos \varphi ; \\
& E_{1}^{\prime} E_{2 !}^{\prime}= \\
& P_{X}=\chi_{X Y Z} \sin \varphi, \quad P_{Y}=x_{X X Z} \sin \varphi, \quad P_{Z}=x_{Z X Y} \cos \varphi \text {. }
\end{aligned}
$$

Note that in general each of these four cases leads to a different refractive index $n_{s}$ for the source wave:

$$
n_{S}=\left(n_{1} \omega_{1}+n_{2} \omega_{2}\right) / \omega_{3}
$$

The wave equation can be solved separately for $s$ - and p-polarized waves $[12,65]$. In the general case a reflected wave $E^{R}$ and a transmitted wave $E^{\mathbf{T}}$ will result. The problem has been discussed extensively by Bloembergen and Pershan [65] and by Jerphagnon and Kurtz [12]. Both articles handle the boundary condition with slightly different assimptions about multiple reflections, interference with backscattered waves and finite beam-size corrections. Here it is only necessary to note that the generated waves can be written in the form:

$$
\mathscr{E}_{L}=f_{Y} P_{Y}, \mathscr{E}_{n}=f_{X} P_{X}+f_{Z} P_{Z}
$$

The factors $f$ depend on the refractive indices at frequency $\omega_{3}$ in the three media $n_{R}, n_{M}, n_{T}$ as well as $n_{S}$ and $\vartheta_{S}$. For the transmitted wave they also depend on the sample thickness. 
It follows that $E_{\mathrm{s}}$ measures $P_{Y}$ while $E_{\mathrm{p}}$ measures a linear combination of $P_{X}$ and $P_{Z}$. This linear combination is in general not the transversal component of $P$, i.e. the component of $P$ perpendicular to $k_{s}$. Unless in the case of normal incidence $\left(\vartheta_{S}=0\right)$ or perfect phasematching $\left(n_{S}=n_{M}\right)$ the longitudinal component of $P_{p}$ will contribute. This is important for the measurement of the "chiral" tensor components $X_{X Y Z}=X_{X Z Y}$, and $X_{Z X Y}$. When $E_{1}, E_{2}$, and $P$ all lie in the same plane, the isotropic part of the tensor $P \otimes E_{\mathrm{t}} \otimes E_{2}$ vanishes:

$$
\begin{aligned}
& \left(P \otimes E_{\mathrm{I}} \otimes E_{2}\right)_{0}^{0} \\
& \quad=(i / \sqrt{6})\left(P_{X} E_{1 Y} E_{2 Z}+P_{Z} E_{1 X} E_{2 Y}+P_{Y} E_{1 Z} E_{2 X}-P_{Z} E_{1 Y} E_{2 X}-F_{Y} E_{1 X} E_{2 Z}-P_{X} E_{1 Z} E_{2 Y}\right)=0
\end{aligned}
$$

Consequently, $x_{u}^{0}$ cannot be measured.

A single measurement is characterized by the polarization of $E_{1}, E_{2}$, and the analysator for the generated wave, each abbreviated by $s$ or p. The experiments (pss), (sps). and (ssp) will yield one tensor element each, namely $x_{z x x}, x_{x z x}$, and $x_{x x z}$. With these $\chi_{z z z}$ is accessible from (ppp). Several solutions for $x z z z$ will be possible depending on the choice of sign for the other three tensor components. This problem can be resolved when data obtained for several angles of incidence or from both reflection and transmission are included into the analysis [52]. The three chiral components are accessible with the experiments (spp), (psp), and (pps). When the transversal component of $P$ does not contribute, $f_{Z} / j_{X}=$ $\tan \varphi$ and the three measurementc become linearly dependent. This leads to the same conclusion as drawn earlier from eq- (67).

\section{Conclusion}

The non-linear optical susceptibility $x^{(2)}$ of a rotationally invariant sample can have up to seven independent components. A method to determine these in sum- or difference-frequency mixing experiments has been outlined. The macroscopic tensor elements are linked to the tensor elements of the molecular hyperpolarizability $\beta$ through an ensemble averaged with an orientational distribution function. In cases where this probability function is known by design, e.g. through orientation of molecular dipoies in a static electric field, up to seven components of $\beta$ can be found. Such a method has promising aspects for the study of organic non-linear optical materials, especially when the molecules of interest form centrosymmetric crystals. On the other hand, when the dominant molecular tensor elements are known, orientational information can be obtained. This has especially interesting applications for surface studies by second-order non-linear optical effects which are beeing developed during recent years.

\section{Peferences}

[1] D.1. Williams, ed. Nonlinear optical properties of crganic and polymer materials, A.CS. Symp. Ser. 233 (1983).

[2] K Jain. J.I. Crowley. G.I. Hewig. Y.Y. Cheng and R.J. Twieg. OpL Laser Toch. 13 (1981) 297.

[3] P.D. Southgate and D.S. Hall. Appl Phys. Letters 18 (1971) 456.

[4] K. Kato, IEEE J. Quantum Electron. QE-16 (1980) 1288.

[5] S. Ayers, M.M. Faktor, D. Marr and J.L. Stevenson. J. Mater. Sci. 7 (1971) 31.

[G] J.L. Stcrenson. J. Phys. D 6 (1973) L13.

[7] G.H. Hewig aad K. Jain, OpL Commun 47 (1983) 347.

[8] P.D. Maker. R.W. Terhune M. Nisenoff and CM. Savage Phys. Rev. Letters 8 (1962) 21.

[9] S.K. Kurze and T.T. Perry, J. Appl. Phys. 39 (1968) 3798.

[10] G.D. Boyd and D.A. Kleinman. J. Appl Phys. 39 (1968) 3597.

[II] J.J. Wynne and N. Bloembergen. Phys. Rev. 183 (1969) 1211.

[12] J. Jerphagnon and S.K. Kurt. J. Appl. Phys. 41 (1970) 1667. 
[13] B.F. Levine, CG. Bethea, C.D. Thurmond, R.T. Lynch and J.L Bernstein, J. Appl. Phys. 50 (1979) 2523.

[14] G.R Meredith, B. Buchalter and C. Hanzlik, J. Chem. Phys. 78 (1983) 1533.

[15] G.R Meredith, B. Buchniter and C Hanzlil, J. Chem. Phys- 78 (1983) 1543.

[16] G.R Meredith and B. Buchalter, J. Chem. Phys 78 (1983) 1615.

I17] G.R. Meredith and B. Buchaiter, J. Chem. Phys. 78 (1983) 1938.

[18] N. Blocmbergen, Nonlinear optics (Benjamin, New York, 1965).

[19] B.F. Levine, Chem. Phys. Letiers 37 (1976) 516.

[20] J_A Giordmaine, Phys. Rev. 138A (1965) 1599.

[21] B. Dick and R.M. Hochstrasser, Phys. Rev. Letters 51 (1983) 2221.

[22] B. Dick and R.M. Hochstrasser, Chem. Phys. 91 (1984) 1.

[23] R.W. Terhume P.D. Maker and CM. Savage. Phys. Rev. Letters 8 (1962) 404.

[24] P.D. Maker and R.W. Terhune, Phys. Res. 137A (1965) 801.

[25] G. Mayer, Compl. Rend 267B (1968) 54.

[26] J. Jerphagnon, Phys. Rev. B2 (1970) 1091.

[27] G. Hauchecome, F. Kerherve and G. Mayer, J. Phys. (Paris) 32 (1971) 47.

[28] B.F. Levine and C.G. Beihea, Appl. Phys. Letters 24 (1974) 445:

[29] B.F. Levine and CG. Bethea, I. Chem. Phys. 60 (1974) 3856.

[30] B.F. Levine and C.G. Bethea, J. Chem. Phys 63 (1975) 2666.

[31I B.F. Levine and C.G. Bethea, J. Chem. Phys. 65 (1976) 1989.

[32] B.F. Levine and C.G. Bethea, J. Chem. Phys. 65 (1976) 2429.

(33) B.F. Levine and CG. Bethea, J. Chem. Phys. 66 (1977) 1070.

[34] B.F. Levine J. Chem. Phys. 63 (1975) 115.

[35] B.F. Levine Chem. Ptys. Letters 37 (1976) 516.

[36] C.G. Bethea, Appl. Opt. 14 (1975) 1447.

[37] CG. Bethea, J. Chem. Phys. 69 (1978) 1312.

[38] J.L. Oudar and D.S. Chemla, Opt Commun. 13 (1975) 164.

[39] J.L Oudar and H. LePerson, Opt. Commun. 15 (1975) 258.

[40) D.S. Chemla J.L. Oudar and J. Jerphagnon. Phys Rev. B12 (1975) 4534.

[41] I.L Oudar, J. Chem. Phys. 67 (1977) 446

[42] J.L Oudar. D.S. Chemla and E Batifol, J. Chem. Phys. 67 (1977) 1626.

[43] S. Kielich, IEEE J. Quantum Electron. QE-S (1969) 562.

[44] T.F. Heinz CK. Chen, D. Ricard and Y.R. Shen. Phys. Rev. Letters 48 (1982) 478.

[45] T.F. Heinz, H.W.K. Tom and Y.R. Shen, Phys. Rev. A28 (1983) 1883.

[46] F. Brown and M. Matsuoka Phys. Rev.-185 (1969) 985.

[47] J.M. Chen, J.R. Borer. CS. Wang and C.H. Lee Opt. Commun. 9 (1973) 132

[48] C.K. Chen, T.F. Heinz D. Ricard and Y.R. Shen, Phys. Rev. Letters 46 (i981) 1010.

[49] T.F. Heinz, C.K. Chen, D. Ricard and Y.R. Shen, Chem. Phys Letters 83 (1981) 180.

[50] G.A. Reider. A.J. Schmidi and G. Marowsky, Opt Commun 47 (1983) 223.

[51] G. Marowski. A. Gieruiski, G.A. Reider and A.J. Schmidt. Appl Phys B34 (1984) 69.

[52] B. Dick, A. Gierulski, G. Marousky and G.A. Reider, to be published.

[53] EE Havingt and P. van Pelt, Ber. Bunsenges. Physik. Chem. 83 (1979) 816.

[54] EE Havinga and P. van Pelt. Mol. Cryst-Liquid Cryst. 52 (1979) 449.

[55I P.D. Maker. Phys Rev. Al (1970) 923 .

[56] J. Jerphagnon. Phys. Rev. B2 (1970) 1091.

[57] B.L Siver, Irreducible tensor methods (Academic Press, New York, 1976).

[58] A.R. Edmonds, Angular momentum in quantum mechanies (Princeton Univ. Press, Princeton, 1974).

[S9] M.E Rose, Elementary theory of angular momentum (Whley, New York, 1957).

[60] A.A. Wolf. Am. J. Phys. 37 (1969) 531.

[61] P.R. Monson and W.M. McClain, J. Chem. Phys. 53 (1970) 29.

[62] W.M. McClain, J. Chem. Phys 55 (1971) 2789.

[63] P.R. Monson and W.M. McClain, J. Chem. Phys. 56 (1972) 4817.

[64] S. Lalama and A.F. Garito, Phys Rev. A20 (1979) 1179.

[65] N. Bloembergen and P.S. Pershan, Phys. Rev. 128 (1962) 606. 